\title{
Development of Extragenital Lichen Sclerosus in Malignant Melanoma Patients Treated With Ipilimumab in Combination With Nivolumab
}

\author{
S. Morteza Seyed Jafari*, Laurence Feldmeyer and Robert E. Hunger \\ Department of Dermatology, Inselspital, Bern University Hospital, University of Bern, Bern, Switzerland
}

The immune checkpoint inhibitors opened a new era for the treatment of melanoma. Nowadays, combined immune checkpoint inhibitors are administered to provide additive or synergistic effects on anti-melanoma immunity. The use of these drugs comes with serious adverse events related to excessive immune activation. Here, we present development of extragenital lichen sclerosus in a patient with metastatic malignant melanoma, during the combined therapy with checkpoint inhibitors.

Edited by: Nihal Ahmad,

University of Wisconsin-Madison,

United States

Reviewed by:

Thilo Gambichler, University Hospital of the Ruhr,

Germany

Jasmine George,

Medical College of Wisconsin,

United States

*Correspondence:

S. Morteza Seyed Jafari

seyedjafarism@yahoo.com

Specialty section:

This article was submitted to

Skin Cancer,

a section of the journal

Frontiers in Oncology

Received: 17 June 2020

Accepted: 25 August 2020

Published: 07 October 2020

Keywords: checkpoint inhibitors, extragenital lichen sclerosus et atrophicus, ipilimumab, malignant melanoma, metastatic, nivolumab

\section{INTRODUCTION}

The immune checkpoint inhibitors opened a new era for the treatment of melanoma (1). As potent immune checkpoint inhibitors, antibodies against cytotoxic T-lymphocyte-associated protein 4 (CTLA-4) and programmed cell death protein 1 (PD-1) are currently in clinical use worldwide (1). Ipilimumab, a human IgG1 monoclonal antibody, blocks CTLA-4 (1). Nivolumab, a fully human IgG4 anti-PD-1 antibody, blocks selectively the interaction of PD-1 and PD-L1/PD-L2 (1). Nowadays, combined immune checkpoint inhibitors are administered to provide additive or synergistic effects on anti-melanoma immunity (1). The use of these drugs comes with serious adverse events related to excessive immune activation, collectively known as immune-related adverse events, such as colitis, hepatitis, thyroiditis, lichenoid dermatoses, bullous dermatoses, psoriasis, and morphea $(2,3)$. The recent papers by Wernham et al. (3) and di Meo et al. (2) described an association between nivolumab treatment and genital and extragenital lichen sclerosus, respectively. However, there are no cases in the literature on the association between combined immune checkpoint inhibitors and extragenital lichen sclerosus. Here, we present development of extragenital lichen sclerosus in a patient with metastatic malignant melanoma, during the combined therapy with checkpoint inhibitors.

Citation:

Seyed Jafari SM, Feldmeyer $L$ and Hunger RE (2020) Development of Extragenital Lichen Sclerosus in Malignant Melanoma Patients Treated With Ipilimumab in Combination With Nivolumab.

\section{REPORT OF THE CASES}

A primary melanoma with $0.6-\mathrm{mm}$ Breslow depth and without ulceration was detected on the back of a 39-year-old female patient. Thereafter, the patient underwent regular clinical follow-up. Six years later, during a routine control, metastatic lesions in the brain and lung were diagnosed. Therefore, a combined therapy with ipilimumab and nivolumab was initially started and followed by monotherapy with nivolumab, as discussed before (4). The therapy was stopped after 2 years 


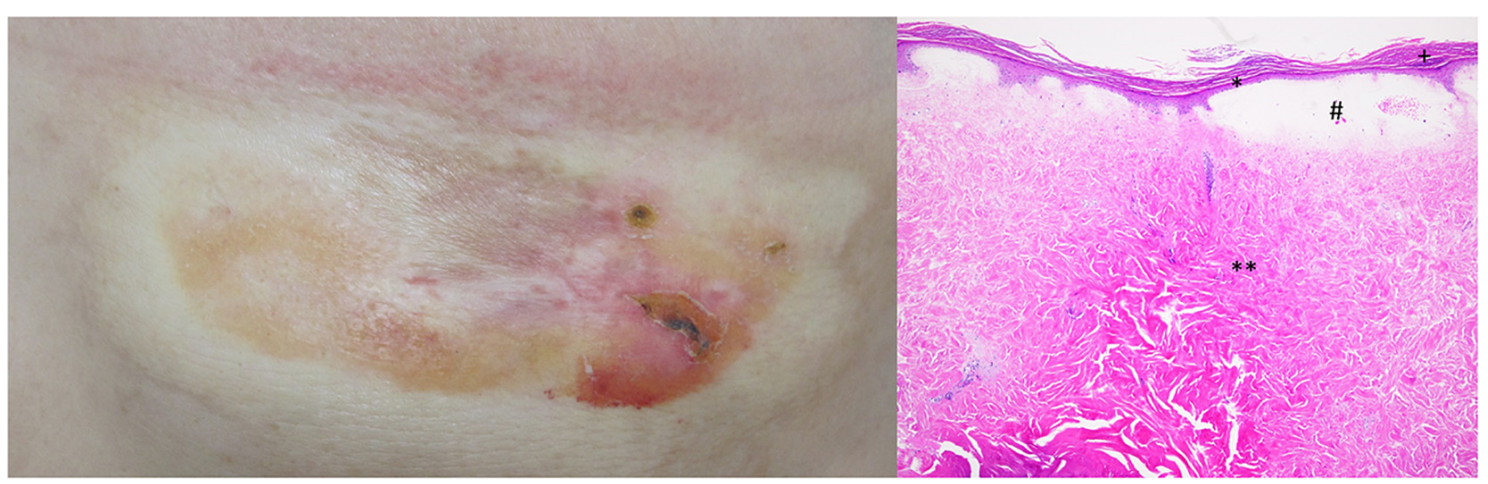

FIGURE 1 | Clinical image shows an atrophic, indurated, and hypopigmented plaque on left submammary region. Histologically, extragenital lichen sclerosus is characterized by hyperkeratosis (+), epidermal atrophy with flattening of rete ridges $\left(^{*}\right)$, edema in the superficial dermis (\#), and homogenized collagen in the dermis with subtotal loss of skin adnexal structures $\left({ }^{* \star}\right)$.

with acceptable clinical response. However, during the last months of the therapy, she presented with an itchy atrophic, indurated hypopigmented plaque on left submammary region (Figure 1). The clinical suspicion of extragenital lichen sclerosus was confirmed by histological evaluation of the lesion revealing hyperkeratosis, epidermal atrophy with flattening of rete ridges, edema in the superficial dermis, and homogenized collagen in the dermis with subtotal loss of skin adnexal structures (Figure 1). Therefore, a local therapy with clobetasol propionate $0.05 \%$ cream was initiated. After 2 weeks of daily application, the therapy was reduced to twice weekly, and a daily therapy with tacrolimus ointment $0.1 \%$ was added. This combination therapy significantly improved the patients' itch and induration of the plaque.

\section{DISCUSSION}

To our knowledge, our case is the first report of an association between extragenital lichen sclerosus during the combined therapy with checkpoint inhibitors. Similar to the cases reported by Wernham et al. (3) and di Meo et al. (2), our patient presented with an itchy atrophic, indurated hypopigmented plaque during the therapy. The etiology of lichen sclerosus, a chronic inflammatory connective tissue disorder, has not been fully elucidated $(3,5-7)$. However, the increased prevalence of organ-specific autoantibodies in lichen sclerosus, such as extracellular matrix protein 1, supports that the autoimmune mechanisms might play a pathogenetic role $(5,6,8)$. In addition, increased levels of Th1-specific cytokines, dense T-cell infiltrates, and enhanced BIC/miR-155 expression has been found $(2,3$, 7). This might explain an association of lichen sclerosus with checkpoint inhibitor therapy. As ipilimumab increases activated $T$ cells and enhances humoral immunity and nivolumab prevents the deactivation of $\mathrm{T}$ lymphocytes, thus promoting self-reactive $\mathrm{T}$ cells $(2,9)$, both drugs might lead to autoimmune diseases, such as lichen sclerosus, as reported before $(2,3)$.
In conclusion, extragenital lichen sclerosus should be considered as one of the immune-related adverse events of combined therapy with checkpoint inhibitors. Local therapy with corticosteroids and/or calcineurin inhibitors could reduce the patients' discomfort and avoid disease progression. Despite presence of strong clinical and histological clues in the patients, occurrence of extragenital lichen sclerosus irrelevant to the immune therapy could not be excluded. Therefore, further studies might be demanded to investigate this issue in more details.

\section{DATA AVAILABILITY STATEMENT}

The raw data supporting the conclusions of this manuscript will be made available by the authors, without undue reservation, to any qualified researcher.

\section{ETHICS STATEMENT}

Ethical review and approval was not required for the study on human participants in accordance with the local legislation and institutional requirements. The patients/participants provided their written informed consent to participate in this study.

\section{AUTHOR CONTRIBUTIONS}

SS, LF, and RH designed the study and performed acquisition, analysis, and interpretation of data, performed critical revision of the manuscript for important intellectual content. SS wrote the manuscript. All authors contributed to the article and approved the submitted version.

\section{FUNDING}

This work was supported by Department of Dermatology, Bern University, Bern, Switzerland. 


\section{REFERENCES}

1. Furue M, Ito T, Wada N, Wada M, Kadono T, Uchi H. Melanoma and immune checkpoint inhibitors. Curr Oncol Rep. (2018) 20:29.

2. di Meo N, Conforti C, Corneli P, Vezzoni R, Retrosi C, Pizzichetta MA, et al. Nivolumab-associated extragenital lichen sclerosus et atrophicus. Clin Exp Dermatol. (2020) 45:350-2.

3. Wernham AGH, Shah F, Velangi S. Nivolumab PD-1 inhibitor immunotherapy associated with vulvar, perineal and perianal lichen sclerosus. Clin Exp Dermatol. (2019) 44:e22-3. doi: 10.1111/ced.13825

4. Larkin J, Chiarion-Sileni V, Gonzalez R, Grob JJ, Cowey CL, Lao CD, et al. Combined nivolumab and ipilimumab or monotherapy in untreated melanoma. N Engl J Med. (2015) 373:23-34.

5. Kim YG, Lee MW, Shin JM, Jeong MG, Ko JY. Colocalization of nonsegmental vitiligo and extragenital lichen sclerosus in a 45-year-old female patient with Hashimoto's thyroiditis. J Dermatol. (2015) 42:333-4. doi: 10.1111/1346-8138. 12779

6. Fistarol SK, Itin PH. Diagnosis and treatment of lichen sclerosus: an update. Am J Clin Dermatol. (2013) 14:27-47. doi: 10.1007/s40257-012-0006-4

7. Terlou A, Santegoets LA, van der Meijden WI, Heijmans-Antonissen C, Swagemakers SM, van der Spek PJ, et al. An autoimmune phenotype in vulvar lichen sclerosus and lichen planus: a Th1 response and high levels of microRNA-155. J Invest Dermatol. (2012) 132(Pt 1): 658-66.

8. Gambichler T, Skrygan M, Czempiel V, Tigges C, Kobus S, Meier JJ, et al. Differential expression of connective tissue growth factor and extracellular matrix proteins in lichen sclerosus. J Eur Acad Dermatol Venereol. (2012) 26:207-12. doi: 10.1111/j.1468-3083.2011.04037.x

9. Berraondo P. Mechanisms of action for different checkpoint inhibitors. HemaSphere (2019) 3:28-30. doi: 10.1097/hs9.0000000000000244

Conflict of Interest: The authors declare that the research was conducted in the absence of any commercial or financial relationships that could be construed as a potential conflict of interest.

Copyright (C) 2020 Seyed Jafari, Feldmeyer and Hunger. This is an open-access article distributed under the terms of the Creative Commons Attribution License (CC BY). The use, distribution or reproduction in other forums is permitted, provided the original author(s) and the copyright owner(s) are credited and that the original publication in this journal is cited, in accordance with accepted academic practice. No use, distribution or reproduction is permitted which does not comply with these terms. 